University of Massachusetts Amherst

From the SelectedWorks of Vincent Rotello

February 16, 2010

Chemically Directed Immobilization of Nanoparticles onto Gold Substrates for Orthogonal Assembly Using Dithiocarbamate Bond Formation

\author{
MH Park \\ XX Duan \\ Y Ofir \\ B Creran \\ D Patra, et al.
}




\title{
Chemically Directed Immobilization of Nanoparticles onto Gold Substrates for Orthogonal Assembly Using Dithiocarbamate Bond Formation
}

\author{
Myoung-Hwan Park, ${ }^{\dagger}$ Xuexin Duan, ${ }^{\ddagger}$ Yuval Ofir, ${ }^{\dagger}$ Brian Creran, ${ }^{\dagger}$ Debabrata Patra, ${ }^{\dagger}$ \\ Xing Yi Ling, ${ }^{\ddagger}$ Jurriaan Huskens, ${ }^{*},{ }^{\ddagger}$ and Vincent M. Rotello*, ${ }^{\dagger}$
}

Department of Chemistry, University of Massachusetts, Amherst, Massachusetts 01003, and Molecular Nanofabrication \& Supramolecular Chemistry and Technology Groups, University of Twente,

7500 AE Enschede, The Netherlands

\begin{abstract}
Dithiocarbamate-mediated bond formation combined with soft lithography was used for the selective immobilization of amine-functionalized silica nanoparticles on gold substrates. The available amine groups on the upper surface of the immobilized silica nanoparticles were further utilized for postdeposition of additional materials including particles, dyes, and biomolecules. The robustness of dithiocarbamate-mediated immobilization enables orthogonal assembly on surfaces via selective removal of the masking thiol ligands using iodine vapor etching followed by further functionalization.
\end{abstract}

KEYWORDS: dithiocarbamate $\bullet$ orthogonal assembly $\bullet$ nanoparticles $\bullet$ immobilization $\bullet$ iodine etching

\section{INTRODUCTION}

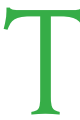
he unique electronic, magnetic, and optical properties
of nanoparticles (NPs) $(1,2)$ make them useful build-
ing blocks for nanodevices and biofabrication $(3-5)$. Site-selective adsorption/immobilization of NPs on surfaces at desired positions is a key fabrication step for these applications. Various patterning strategies have been developed using both covalent bonding and noncovalent interactions (6-13). Gold substrates are promising materials that can be used to construct protein patterns and DNA assemblies for biosensors (14-16) as well as useful platforms for surface plasmon resonance imaging techniques (17). However, alkanethiol-based chemistries have been generally used for the chemisorptions of NPs on gold surfaces $(18,19)$, and these assemblies are sensitive to oxidation, disassociation, thermal desorption, and photochemical degradation (20-24).

Recently, we reported a dithiocarbamate (DTC)-based approach for immobilization of thiophilic metal and semiconductor NPs on amine-functionalized self-assembled monolayers (SAMs) anchored to silica surfaces (25-27). This versatile technique provides systems with excellent stability to organic solvents and high ionic strength (28-30). Here, we extend this technique to the attachment of silica NPs functionalized with amine groups $\left(\mathrm{SiO}_{2} \mathrm{NPs}\right)$ onto gold

* Corresponding author. E-mail: rotello@chem.umass.edu (V. M. R.); J.Huskens@tnw.utwente.nl (J. H.). Tel.: (413) 545-2058 (V. M. R.);

31-53489-2995 (J. H.). Fax: (413) 545-4490 (V. M. R.); 31-53489-4645 (J. H.)

Received for review December 16, 2009 and accepted February 8, 2010

${ }^{\dagger}$ University of Massachusetts

* University of Twente.

DOI: $10.1021 / \mathrm{am} 9009007$

• 2010 American Chemical Society substrates. This method generates a scaffold with dense functionalization and high surface area for immobilization of NPs and biomolecules. These assemblies on metal surfaces provide an efficient strategy for the construction of biosensors and electroluminescent devices $(31-35)$. One of the key fabrication steps is to pattern NPs and biomolecules orthogonally for these types of applications, but the usage of thiol chemistry has limited the creation of orthogonal assemblies because of the labile binding of alkanethiols to gold surfaces. In contrast, the robustness of the DTCmediated assembly technique provides a more reliable means for creating orthogonal assemblies on the substrates.

As a first example, amine-fuctionalized $\mathrm{SiO}_{2} \mathrm{NPs}$ were immobilized on gold substrates using DTC bond formation in a controlled fashion, with soft lithography utilized for octadecanethiol (ODT) patterning that serves as a mask. Amine functional groups on $\mathrm{SiO}_{2}$ NPs were converted to DTCs in the presence of $\mathrm{CS}_{2}$, affording in situ immobilization of NPs onto gold substrates. Available amine groups on the upper surface of the $\mathrm{SiO}_{2}$ NPs were then used for further chemical reactions, as demonstrated by electrostatic deposition of fluorescent beads/biomolecules and covalent attachment of organic dyes and quantum dots (QDs). Moreover, the strong and multivalent binding of DTC-mediated NP immobilization on surfaces makes it possible to selectively remove the chemical mask of thiol ligands during iodine vapor etching and provides a simple and effective tool for the creation of orthogonal assemblies using sequential depositions.

\section{EXPERIMENTAL SECTION}

Materials and Instruments. Au-coated substrates $(1000 \AA$ gold surface thickness) on a silicon wafer (4 in. diameter) were 
Scheme 1. Schematic Illustrations of (a) DTC-Mediated Immobilization of Amine-Functionalized $\mathrm{SiO}_{2} \mathrm{NPs}_{\text {on }}$ Gold Substrates Using $\mu \mathrm{CP}$ and (b) Postdeposition of Fluorescent Materials of PS Beads, BSA, Lissamine, and QD-TOH on the Available Amine Groups on the Upper Surface of $\mathrm{SiO}_{2} \mathrm{NPs}$

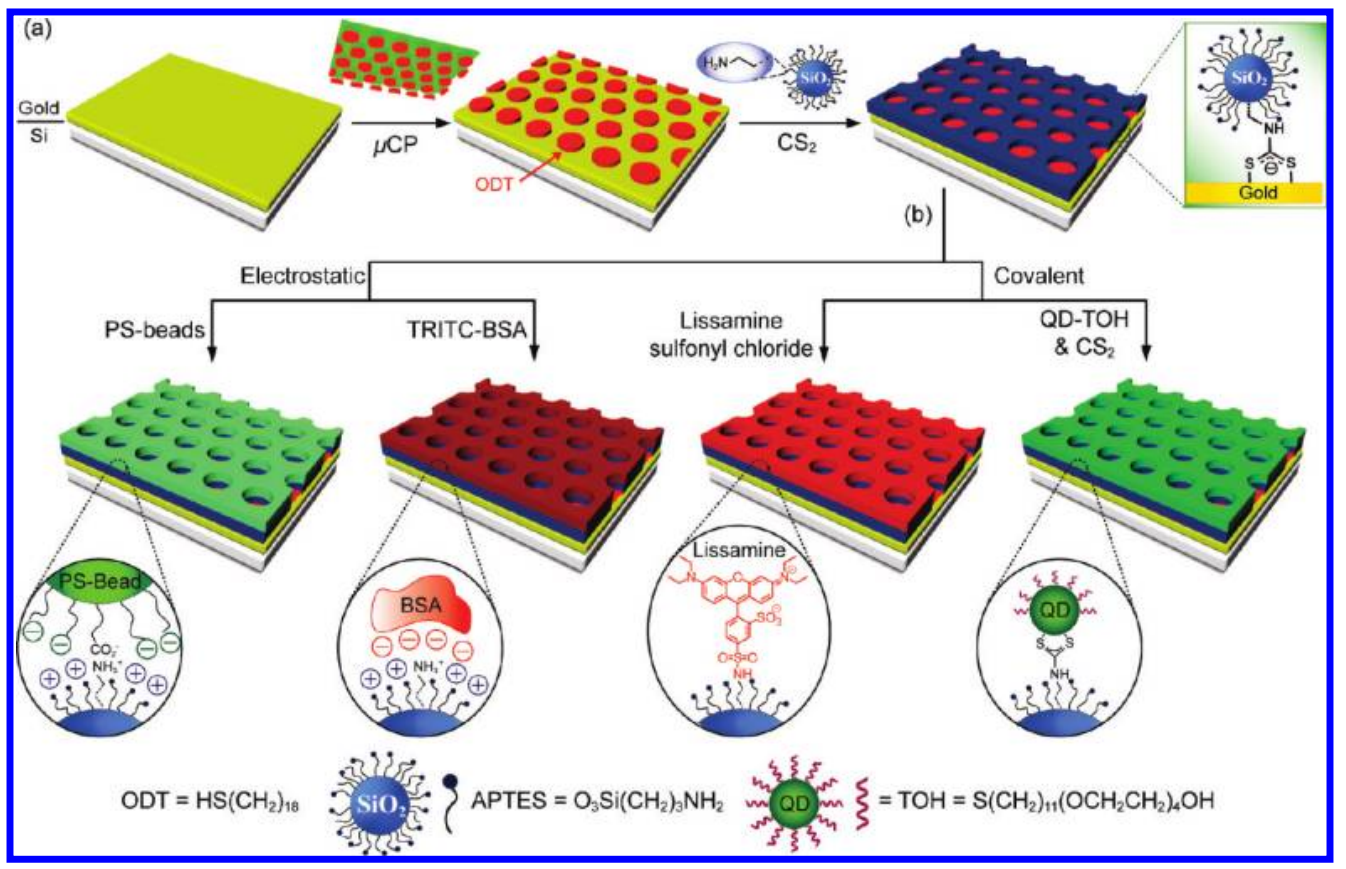

purchased from Aldrich and cleaned just prior to use by immersion in freshly prepared piranha solution (3:1 volume ratio of conc. $\mathrm{H}_{2} \mathrm{SO}_{4}: 30 \% \mathrm{H}_{2} \mathrm{O}_{2}$ ). Caution! Piranha solutions should be handled with great care in open containers in a fume hood. Piranha solutions are highly corrosive, toxic, and potentially explosive. ODT, 3-amino propyl triethoxysilane (APTES), tetraethyl orthosilicate, ammonium hydroxide, iodine, and FITC-BSA (fluorescein isothiocyanate-labeled bovine serum albumin) were also purchased from Aldrich and used as received. Lissamine dye (lissamine rhodamine B sulfonyl chloride) and TRITC-BSA (tetramethylrhodamine isothiocyanate-labeled BSA) were purchased from Invitrogen. The stamp material, Sylgard-184 (poly(dimethylsiloxane, PDMS) and carboxylate-functionalized fluorescent latex beads (PS beads, $\sim 50 \mathrm{~nm}$ ), were obtained from Dow Corning and Polysciences, Inc., respectively. The fluorescent and optical microscopy images were achieved using an Olympus IX51 microscope and Zeiss LSM510 meta confocal microscope. The surface topography of monolayers on the surface was imaged by AFM (Digital Instruments, Nanoscope III) in tapping mode.

Preparation of Amine-Functionalized $\mathrm{SiO}_{2} \quad \mathrm{NPs}$ and QD-TOH. Silica particles ( $\sim 60 \mathrm{~nm})$ with silanol surface groups were prepared using a modified Stöber method, as reported previously $(36,37)$, and functionalized with APTES (see the Supporting Information for detailed procedures). QD-TOH ( 3 $\mathrm{nm})$ was prepared using the same procedure with the previous paper (25).

Preparation of ODT Prepatterned Surfaces by $\mu$ CP. A PDMS stamp was made using a standard technique (38). A PDMS stamp was inked with $2 \mathrm{mM}$ solution of ODT in ethanol. After drying with a nitrogen stream, the stamp was placed manually on the gold substrate to achieve the conformal contact between the stamp and substrate. After $1 \mathrm{~min}$, the stamp was carefully removed and the surface was washed with ethanol and dried under a nitrogen stream.

DTC-Mediated NP Immobilization on Prepatterned ODT Surfaces. The immobilization of $\mathrm{SiO}_{2}$ NPs on uncovered gold areas was achieved by vertically soaking into a mixture of $\mathrm{CS}_{2}$ $(25 \mu \mathrm{L})$ and $\mathrm{SiO}_{2}$ NPS $(0.5 \mathrm{~mL}, 5 \mathrm{mg} / \mathrm{mL})$ in methanol $(3 \mathrm{~mL})$ with $\mathrm{Et}_{3} \mathrm{~N}(25 \mu \mathrm{L})$ for $1 \mathrm{~h}$. The substrates were then removed, rinsed with ethanol and water several times, and finally dried under a nitrogen stream.

Postdeposition of Fluorescent Molecules. Once $\mathrm{SiO}_{2} \mathrm{NPS}$ were captured on gold substrates, the patterned substrates were simply rinsed with a $0.1 \mathrm{M} \mathrm{HCl}$ solution for protonation of amine groups for electrostatic deposition. Subsequently, the substrates were incubated with fluorescent molecules, PS beads $(5 \mathrm{mg} /$ $\mathrm{mL}$ ), and FITC/TRITC-BSA (1 $\mathrm{mg} / \mathrm{mL})$ in water for electrostatic assembly or QD-TOH $(1 \mathrm{mg} / \mathrm{mL})$ in methanol in the presence of $\mathrm{CS}_{2}$ for DTC bond formation, or reacted with Lissamine (1 $\mathrm{mg} / \mathrm{mL}$ ) in acetonitrile with $\mathrm{Et}_{3} \mathrm{~N}$ for sulfonamide reaction. After $1 \mathrm{~h}$, the substrates were washed with ethanol and water several times and then dried under a nitrogen stream.

Iodine Etching of ODT Layers. The substrate with $\mathrm{SiO}_{2} \mathrm{NPs}$ and Lissamine was exposed to a saturated atmosphere of iodine vapor at room temperature for $6 \mathrm{~h}$, followed by washing with ethanol and drying under a nitrogen stream.

\section{RESULTS AND DISCUSSION}

Alkanethiol-based chemistries have been generally used for the chemisorptions of NPs on gold substrates, providing several advantages such as simple processing and easy manipulating using surface place exchange reactions. However, thiol chemistry has been shown to be sensitive to oxidation and disassociation, limiting its usage in the construction of orthogonal assemblies. Conversely, the robustness of the DTC-mediated assembly provides a more reliable way for creating these assemblies on gold surfaces. To demonstrate this application, we immobilized amine-functionalized $\mathrm{SiO}_{2}$ NPS onto gold substrates using DTC bond formation to generate a robust scaffold. In the first experiment, ODT patterns acting as masks were introduced onto gold substrates using $\mu \mathrm{CP}(39,40)$ with PDMS stamps (Scheme $1 \mathrm{a})$. Amine-functionalized $\mathrm{SiO}_{2}$ NPs $(\sim 60 \mathrm{~nm}$ diameter) were anchored to the surface via DTC bond formation in the presence of $\mathrm{CS}_{2}$, affording in situ immobilization 

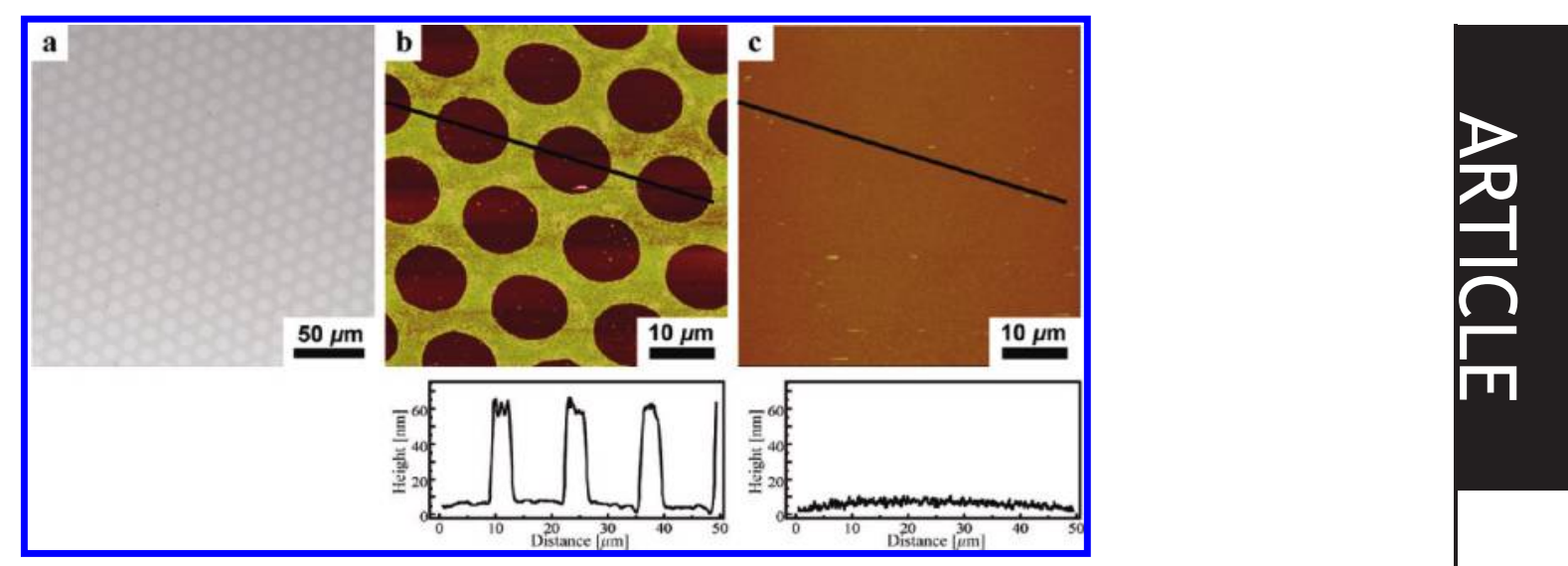

FIGURE 1. (a) Bright-field OM and (b) AFM height images of DTC-mediated immobilization of amine-functionalized silica NP monolayers on gold substrates prepatterned with ODT masks via $\mu \mathrm{CP}$. (c) AFM height image of a control experiment in the absence of $\mathrm{CS}_{2}$.

of NPs. That is, attachment of NPs was performed by immersing the substrate into a solution of $\mathrm{CS}_{2}$ and $\mathrm{SiO}_{2} \mathrm{NPs}$, causing the primary amines on the NPs to react with the $\mathrm{CS}_{2}$ forming DTC groups. These DTC groups effectively hold NPs to the gold surface.

Optical microscopy (OM) and atomic force microscopy (AFM) were utilized for demonstrating the successful attachment of $\mathrm{SiO}_{2} \mathrm{NPs}$ on the exposed gold areas. The bright-field OM image (Figure 1a) indicates the selective deposition of the $\mathrm{SiO}_{2} \mathrm{NPs}$ in large areas. The darker areas show $\mathrm{SiO}_{2} \mathrm{NPs}$ while the brighter regions indicate the ODT that serves as an effective mask for the surface deposition. Figure $1 \mathrm{~b}$ shows AFM height image and line profile image that is in agreement with the height of a monolayer of $\mathrm{SiO}_{2}$ NPs $(\sim 60 \mathrm{~nm})$. Clearly, $\mathrm{SiO}_{2}$ NPs were specifically adsorbed onto the uncovered gold areas; relative to the ODT regions. A control experiment done in the absence of $\mathrm{CS}_{2}$ shows only minimal adsorption of NPs (Figure 1c).

To demonstrate the applicability of this technique for presenting other functionalities, the available amine groups on the upper surface of the immobilization of $\mathrm{SiO}_{2} \mathrm{NPs}$ (41) with high surface area were utilized for specific deposition of fluorescent materials using both supramolecular assembly and covalent binding (Scheme 1b). We first explored the electrostatic assembly of negatively charged PS beads ( $~ 50$ $\mathrm{nm}$ ) onto the available amine groups on the upper surface of the $\mathrm{SiO}_{2}$ NPs. After pretreatment in an acid solution for protonation of amine groups, the patterned substrate was dipped into the solution of negatively charged PS beads followed by a thorough washing, resulting in specific deposition of PS beads on the upper surface of the $\mathrm{SiO}_{2} \mathrm{NPs}$ (Figure 2b).

In addition, we investigated covalent deposition using sulfonamide and DTC chemistry. For the sulfonamide reaction, the silica NP attached substrate was exposed to a solution of Lisamine organic dyes with triethylamine. Figure $2 \mathrm{c}$ shows a typical fluorescence image of Lissamine deposited onto the substrate. Another strategy for deposition of fluorescent materials is to utilize the DTC chemistry again by immersing into a solution of $\mathrm{QD}-\mathrm{TOH}(\sim 3 \mathrm{~nm})$ in the presence of $\mathrm{CS}_{2}$. Fluorescence imaging of the surface also

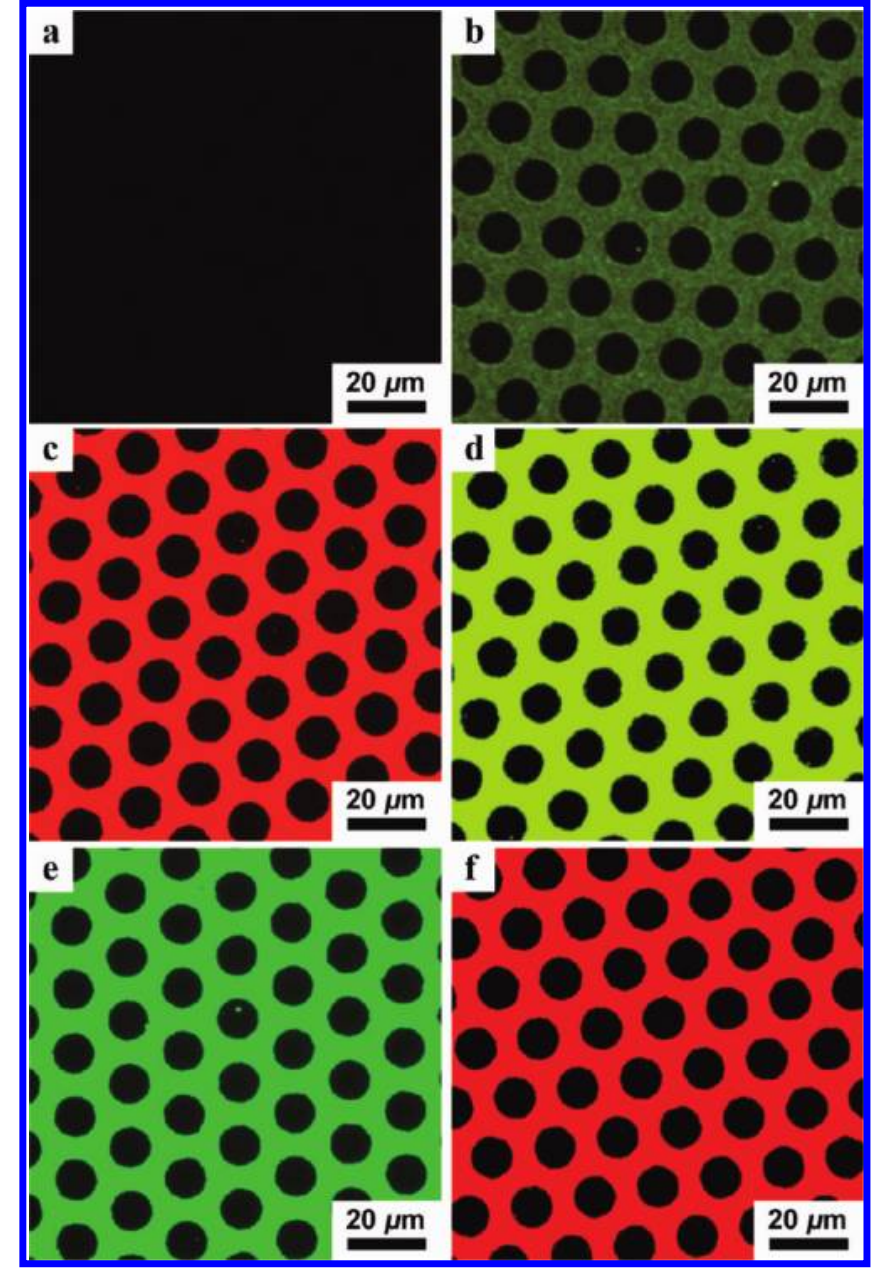

FIGURE 2. Fluorescence OM (a) before and (b) after electrostatic deposition of fluorescent PS beads, (c) sulfonamide reaction with Lissamine, (d) DTC binding of QD-TOH, and electrostatic deposition of biomolecules of (e) FITC-BSA and (f) TRITC-BSA on the upper surface of the immobilized $\mathrm{SiO}_{2} \mathrm{NPs}$ at $480 / 535 \mathrm{~nm}$.

showed successful immobilization of QD-TOH via DTC chemistry (Figure $2 \mathrm{~d}$ ). To further demonstrate the versatility as a scaffold for biofabrication, we explored the electrostatic assembly of biomolecules on the available amine groups. Figure $2 \mathrm{e}, 2 \mathrm{f}$ shows fluorescence images demonstrating the electrostatic deposition of FITC-BSA and TRITC-BSA on the available amine groups, respectively. 
Scheme 2. Schematic Illustration for Selective Removal of ODT Layers and Sequential Deposition of $\mathrm{SiO}_{2}$ NPs and FITC-BSA

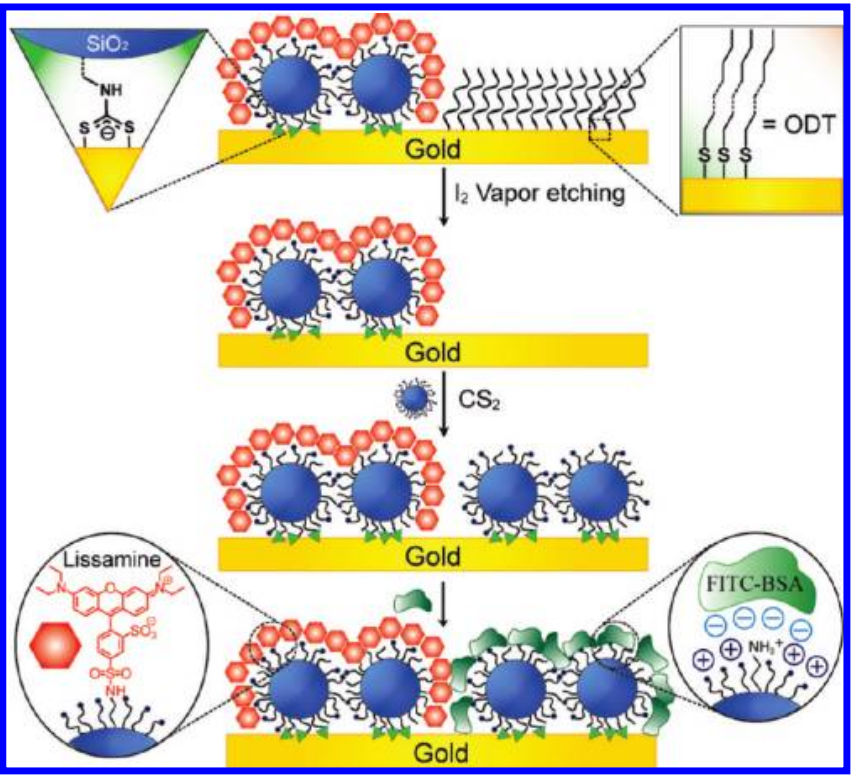

Another ongoing effort in nanodevices and biofabrication is the simultaneous formation of orthogonally assembled patterns onto substrates, i.e. patterns featuring two different components (42-44). The DTC-mediated process provides higher stability than that of thiol chemistry on gold surfaces. Because of the difference in robustness and multivalent attachment of $\mathrm{SiO}_{2}$ NPs on surfaces, the layer of NPs was retained and alkanethiol layers was selectively removed during iodine etching for the creation of an orthogonal assembly. Oxidation of thiol groups to disulfide groups by iodine with concomitant desorption of the SAM is a wellknown reaction (45). In our studies, the patterned substrate with Si NPs and Lissamine dyes was exposed to iodine vapor. The ODT layers were selectively removed, whereas DTC-mediated NP features remained on the gold substrates (Scheme 2). The uncovered bare gold surfaces were then used for immobilization of a second set of $\mathrm{SiO}_{2} \mathrm{NPs}$ using the same DTC-mediated attachment followed by postdeposition of FITC-BSA to prove the selective removal of the ODT layers. The substrate was analyzed using fluorescent $\mathrm{OM}$ and confocal laser scanning microscopy (LSM) to demonstrate these selective orthogonal assemblies (Figure 3 ). The confocal LSM images exhibit the red (>560 nm filter, Figure 3c) and green fluorescent patterns $(475-525 \mathrm{~nm}$ filter, Figure $3 \mathrm{~d}$ ), and a superimposed image of both red and green fluorescent images (Figure 3e).

\section{CONCLUSION}

We have introduced a versatile and reliable technique for capturing NPs on gold substrates based on DTC-mediated assembly. The technique is compatible with soft lithography processes and is effective for postdeposition of other particles/ dyes/biomolecules using the available functional groups on the NPs after the first immobilization. This postdeposition process provides large surface areas with high stability which

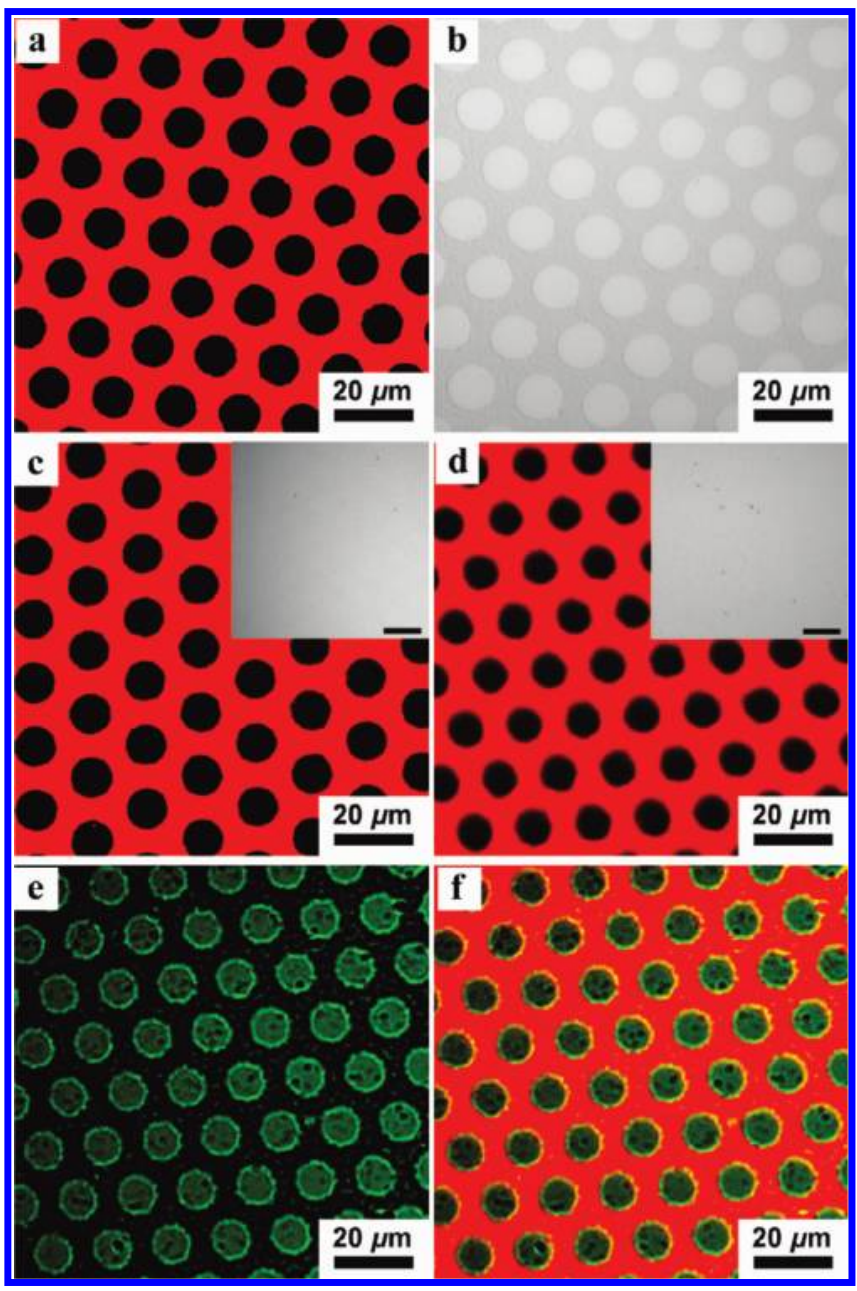

FIGURE 3. (a) Fluorescence and (b) OM images of lissaminefunctionalized surface after iodine vapor etching and (c) after second deposition of $\mathrm{SiO}_{2}$ NPs. (d-f) Confocal LSM images after postdeposition of FITC-BSA excited at $480 \mathrm{~nm}$ using the detection filters of (d) over $560 \mathrm{~nm}$ and (e) $475-525 \mathrm{~nm}$ and (f) a superimposed image of $d$ and e. (Insets are bright-field OM images and scale bars are 20 $\mu \mathrm{m}$.)

can bind fluorescent molecules, increasing their efficiency and sensitivity that can then be applied to various devices involving nanosensors and biosensors. Furthermore, the robustness of DTC-mediated NP assembly makes it available for orthogonal assembly, opening a new way for immobilizing NPs on gold substrates in fields such as nanotechnology and biotechnology.

Acknowledgment. This research was supported by the NSF (CHE-0808945 to V.M.R.), the Center for Hierarchical Manufacturing (NSEC DMI-0531171), MRSEC facilities, and Nanotechnology Network in The Netherlands Nanoned (TMM 7125).

Supporting Information Available: Details of experimental procedures for preparation of amine-functionalized $\mathrm{SiO}_{2}$ NPS and their SEM image and size distribution histogram (PDF). This material is available free of charge via the Internet at http://pubs.acs.org.

\section{REFERENCES AND NOTES}

(1) Sokolova, V.; Epple, M. Angew. Chem. Int. Ed. 2008, 47, $1382-$ 1395. 
(2) Burda, C.; Chen, X.; Narayanan, R.; El-Sayed, M. A. Chem. Rev. 2005, 105, 1025-1102.

(3) Rosi, N. L.; Mirkin, C. A. Chem. Rev. 2005, 105, 1547-1562

(4) Daniel, M.-C.; Astruc, D. Chem. Rev. 2004, 103, 293-346.

(5) Shipway, A. N.; Katz, E.; Willner, I. ChemPhysChem 2000, 1, 18 52 .

(6) Shavel, A.; Gaponik, N.; Eychmüller, A. ChemPhvsChem 2005, 6, $449-451$.

(7) Xu, H.; Hong, R.; Wang, X.; Arvizo, R.; You, C.; Samanta, B.; Patra, D.; Tuominen, M. T.; Rotello, V. M. Adv. Mater. 2007, 19, 1383.

(8) Ofir, Y.; Samanta, B.; Xiao, Q.; Jordan, B. J.; Xu, H.; Arumugam, P.; Arvizo, R.; Tuominen, M. T.; Rotello, V. M. Adv. Mater. 2008, 20, 2561-2566.

(9) Baron, R.; Huang, C.-H.; Bassani, D. M.; Onopriyenko, A.; Zayats, M.; Willner, I. Angew. Chem., Int. Ed. 2005, 44, 4010-4015.

(10) Xu, H.; Hong, R.; Lu, T.; Uzun, O.; Rotello, V. M. I. Am. Chem. Soc. 2006, 128, 3162-3163

(11) Wanunu, M.; Popovitz-Biro, R.; Cohen, H.; Vaskevich, A.; Rubinstein, I. I. Am. Chem. Soc. 2005, 127, 9207-9215.

(12) Crespo-Biel, O.; Dordi, B.; Reinhoudt, D. N.; Huskens, J. I. Am. Chem. Soc. 2005, 127, 7594-7600

(13) Park, S. Y.; Lytton-Jean, A. K. R.; Lee, B.; Weigand, S.; Schatz, G. C.; Mirkin, C. A. Nature 2008, 451, 553-556

(14) Sakata, T.; Maruyama, S.; Ueda, A.; Otsuka, H.; Miyahara, Y. Lanamuir 2007, 23, 2269-2272.

(15) Veiseh, M.; Zareie, M. H.; Zhang, M. Lanqmuir 2002, 18, $6671-$ 6678.

(16) Dandy, D. S.; Wu, P.; Grainger, D. W. Proc. Natl. Acad. Sci. (U.S.A.) 2007, 104, 8223-8228.

(17) Chen, Y.; Nguyen, A.; Niu, L.; Corn, R. M. Lanamuir 2009, 25, $5054-5060$.

(18) Tsuruoka, T.; Takahashi, R.; Nakamura, T.; Fujii, M.; Akamatsu, K.; Nawafune, H. Chem. Commun. 2008, 1641-1643.

(19) Love, J. C.; Estroff, L. A.; Kriebel, J. K.; Nuzzo, R. G.; Whitesides, G. M. Chem. Rev. 2005, 105, 1103-1169.

(20) Flynn, N. T.; Tran, T. N. T.; Cima, M. J.; Langer, R. Lanqmuir 2003, 19, 10909-10915

(21) Schlenoff, J. B.; Li, M.; Ly, H. I.Am. Chem. Soc. 1995, 117, 12528 12536

(22) Aldana, J.; Wang, Y. A.; Peng, X. I. Am. Chem. Soc. 2001, 123, $8844-8850$.

(23) Dasog, M.; Scott, R. W. I. Lanqmuir 2007, 23, 3381-3387.

(24) Ryan, D.; Parviz, B. A.; Linder, V.; Semetey, V.; Sia, S. K.; Su, J.; Mrksich, M.; Whitesides, G. M. Lanamuir 2004, 20, 9080-9088.
(25) Park, M.-H.; Ofir, Y.; Samanta, B.; Arumugam, P.; Miranda, O. R.; Rotello, V. M. Adv. Mater. 2008, 20, 4185-4188.

(26) Park, M.-H.; Ofir, Y.; Samanta, B.; Rotello, V. M. Adv. Mater. 2009, 21, 2323-2327.

(27) Subramani, C.; Ofir, Y.; Patra, D.; Jordan, B. J.; Moran, I. W.; Park, M.-H.; Carter, K. R.; Rotello, V. M. Adv. Funct. Mater. 2009, 19, 2937-2942.

(28) Colorado, R., Jr.; Villazana, R. J.; Lee, T. R. Lanamuir 1998, 14, 6337-6340.

(29) Zhao, Y.; Pérez-Segarra, W.; Shi, Q.; Wei, A. L. Am. Chem. Soc. 2005, 127, 7328-7329

(30) Zhu, H.; Coleman, D. M.; Dehen, C. J.; Geisler, I. M.; Zemlyanov, D.; Chmielewski, J.; Simpson, G. J.; Wei, A. Lanamuir 2008, 24, 8660 - 8666 .

(31) Haes, A. J.; Hall, W. P.; Chang, L.; Klein, W. L.; Duyne, R. P. V. Nano Lett. 2004, 4, 1029-1034.

(32) Willets, A. K.; Duyne, R. P. V. Annu. Rev. Phys. Chem. 2007, 58, 267-297.

(33) Anker, J. N.; Hall, W. P.; Lyandres, O.; Shah, N. C.; Zhao, J.; Duyne, R. P. V. Nat. Mater. 2008, 7, 442-453

(34) Stewart, M. E.; Anderton, C. R.; Thompson, L. B.; Maria, J.; Gray, S. K.; Rogers, J. A.; Nuzzo, R. G. Chem. Rev. 2008, 108, 494-521

(35) Bertoni, C.; Gallardo, D.; Dunn, S. Appl. Phys. Lett. 2007, 90, 034107

(36) Mahalingam, V.; Onclin, S.; Péter, M.; Ravoo, B. J.; Huskens, J.; Reinhoudt, D. N. Lanqmuir 2004, 20, 11756-11762

(37) Hiramatsu, H.; Osterloh, F. E. Lanqmuir 2003, 19, 7003-7011.

(38) Kumar, A.; Biebuyck, H. A.; Whitesides, G. M. Lanamuir 1994, 10, 1498-1511

(39) Xia, Y.; Whitesides, G. M. Angew. Chem. Int. Ed. 1998, 37, 550 575

(40) Gates, B. D.; Xu, Q.; Stewart, M.; Ryan, D.; Willson, C. G.; Whitesides, G. M. Chem. Rev. 2005, 105, 1171-1196.

(41) Park, J.-H.; Gu, L.; Maltzahn, G. v.; Ruoslahti, E.; Bhatia, S. N.; Sailor, M. I. Nat. Mater. 2009, 8, 331-336

(42) Laibinis, P. E.; Hickman, J. J.; Wrighton, M. S.; Whitesides, G. M. Science 1989, 245, 845-847.

(43) Xu, H.; Hong, R.; Lu, T.; Uzun, O.; M., R. V.I. Am. Chem. Soc. 2006, $128,3162-3163$

(44) Ivanisevic, A.; Im, J.-H.; Lee, K.-B.; Park, S.-J.; Demers, L. M.; Watson, K. J.; Mirkin, C. A. I. Am. Chem. Soc. 2001, 123, 12424 12425.

(45) Fan, H.; E, L.; Gabaldon, J.; Wright, A.; Haddad, R. E.; Brinker, C. L. Adv. Mater. 2005, 17, 2587-2590.

AM9009007 